Théologiques

Théologiques

\title{
Aiamie, agir au mieux ? Éthique, rituels catholiques et corps social chez les Anicinabek depuis les années 1950
}

\section{Marie-Pierre Bousquet}

Volume 20, numéro 1-2, 2012

URI : https://id.erudit.org/iderudit/1018865ar

DOI : https://doi.org/10.7202/1018865ar

Aller au sommaire du numéro

\section{Éditeur(s)}

Faculté de théologie et de sciences des religions, Université de Montréal

\section{ISSN}

1188-7109 (imprimé)

1492-1413 (numérique)

Découvrir la revue

\section{Citer cet article}

Bousquet, M.-P. (2012). Aiamie, agir au mieux ? Éthique, rituels catholiques et corps social chez les Anicinabek depuis les années 1950. Théologiques, 20(1-2), 385-417. https://doi.org/10.7202/1018865ar
Résumé de l'article

Cet article analyse la performance de rituels catholiques communautaires, comprenant des processions et des bénédictions, chez les Anicinabek (Algonquins). Il avance que leur étude donne à voir, dans le temps, l'éthique anicinabe. En effet, la période retenue, entre les années 1950 et 2000, correspond à des changements sociaux qui ont bouleversé l'ordre sociocosmique anicinabe. En portant attention à leur déroulement, à ce qu'ils mettent en scène du corps social, des relations avec le clergé et avec le surnaturel, nous postulons que ces rituels révèlent tant les transformations de l'imaginaire social et cosmologique anicinabe qu'un système de pensée pragmatique permettant de prendre les décisions pour se comporter, être et agir au mieux. Enfin, dans le contexte actuel où le catholicisme est en nette régression, nous examinons les nouveaux rites collectifs pour constater que ceux qui promeuvent les « bonnes manières de faire » tiennent essentiellement du domaine politique, terrain où se négocie maintenant la moralité commune. 


\title{
Aiamie ${ }^{1}$, agir au mieux? Éthique, rituels catholiques et corps social chez les Anicinabek depuis les années 1950
}

\author{
Marie-Pierre BousQuET" \\ Anthropologie \\ Université de Montréal (Canada)
}

Les sauvages de la Barrière eux aussi voudraient pouvoir organiser une petite procession. Mais, hélas! Que faire? Et bien, ils ont une petite bannière, représentant l'image de la Sainte Vierge, et nous avons le bonheur d'avoir un pauvre petit ostensoir et un encensoir qu'on jetterait dans quelque coin de vos sacristies. [...] La congrégation s'est rangée sur deux lignes, précédée de leur petite bannière et accompagnant le Très Saint Sacrement en faisant retentir les airs de leurs plus beaux cantiques. (J. P. Gougeon o.m.i. 1880, 227)

Chez les Amérindiens, nous sommes véritablement en pays de missions. [...] On me dit qu'ils sont friands de célébrations, de fêtes à caractère liturgique et social. (Jean Gratton, évêque de Mont-Laurier, Visite pastorale à NotreDame-du-Rosaire, Maniwaki, les 24 et 25 janvier 1981, p. 63, Archives Deschâtelets)

1. Aiamie: il prie, il est du nombre des priants, il est chrétien. Le mot, joint à d'autres, permet de décliner tout le vocabulaire de la religion et pas seulement catholique.

* Marie-Pierre Bousquet est professeur agrégé au Département d'anthropologie de l'Université de Montréal. Ses recherches actuelles portent sur le paysage des croyances religieuses anicinabek (algonquines) depuis le XIX ${ }^{\mathrm{e}}$ siècle et les rôles des missionnaires catholiques dans l'organisation politique et administrative des Affaires indiennes, avec études de cas chez les Anicinabek. 
À partir des années 1920-1930, à part ce que les missionnaires qualifient d'exceptions, les Anicinabek ${ }^{2}$ sont considérés comme étant tous catholiques. Concernant ceux du lac Abitibi, l'ethnologue MacPherson écrit $(1930,112)$ : "The natives today claim that only the power of the Christian Cross is greater than the supernatural ability of the conjuror ». Si les opinions des Anicinabek à ce sujet vont beaucoup changer à partir des années 1960-1970, avec une réhabilitation graduelle du chamanisme, j'ai pu noter, au cours de nombreuses études sur le terrains, effectuées en Abitibi depuis 1996, que mes informateurs se souviennent avec nostalgie des processions, bénédictions et autres fêtes communautaires qui se déroulaient dans un cadre chrétien. Les prêtres, également, avaient remarqué à quel point les Amérindiens aimaient ces célébrations. Il ne semble pas s'agir d'un regret des pratiques catholiques, à l'heure où les églises des villages anicinabek sont presque vides et où plusieurs n'ont plus de curé. Tout le monde aime les fêtes, serions-nous enclins à penser. D'ailleurs, les Anicinabek regrettent autant les carnavals d'antan et autres manifestations collectives d'ordre profane, que leurs ancêtres ou eux-mêmes ont connus et qui ne se déroulent plus toujours de façon régulière, faute d'organisateurs, dans les villages ou sur les territoires. L'aspect festif n'est certainement pas à négliger pour comprendre cet attachement à ces cérémonies religieuses, mais il ne suffit pas à l'expliquer. En effet, une seule de ces occasions de rassemblement encadrées par un contexte catholique subsiste et il ne s'agit guère de moments de réjouissances: je fais ici allusion aux funérailles ${ }^{3}$. Je propose donc, en m'intéressant à quelques-uns de ces rituels qui se déroulaient dans des espaces publics signifiants pour les Anicinabek, de me pencher sur ce qu'ils représentaient du sens de la communauté, ma prémisse de départ étant que «dans n'importe quelle société et moment historique, une des fonctions fondamentales des fêtes [...] a été celle de constituer une occasion rituelle pour la reproduction d'identités; pour établir et reproduire un nous par opposition à eux" (Moreno-Navarro 1986, 446). J'émets l'hypothèse que ces rituels permettent une lecture du modèle social en vigueur chez les Anicinabek, depuis l'incorporation du catholicisme à l'ensemble plus large de leur système de croyances, modèle

2. L'ethnonyme Anicinabe (prononcer Anichinabé; -ek au pluriel) est le mot vernaculaire par lequel les Algonquins se désignent eux-mêmes. Les Anicinabek, au Québec, vivent dans les régions de l'Outaouais et de l'Abitibi-Témiscamingue.

3. D'autres cérémonies catholiques subsistent, constituant des options parmi d'autres pour marquer les rites de passage, comme le baptême et le mariage. Mais il s'agit alors de cérémonies privées. Les funérailles, elles, ne nécessitent pas d'invitations. 
élaboré en réponse au bouleversement de l'ordre sociocosmique anicinabe dû à l'alcoolisme, à la colonisation du territoire, à la baisse des ressources fauniques subséquentes, à la sédentarisation progressive et, paradoxalement à première vue, à l'évangélisation.

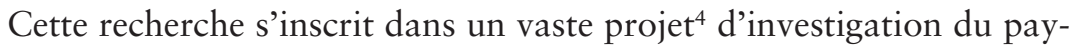
sage des croyances religieuses chez les Anicinabek depuis le XIX ${ }^{\mathrm{e}}$ siècle, avec un intérêt particulier pour le catholicisme populaire (Bousquet 2007; 2008; 2012a ; Bousquet et Morissette 2008). Le recueil d'informations a été effectué à partir de sources orales (témoignages d'aînés et d'individus des générations suivantes ${ }^{5}$, entrevues avec des curés de paroisses amérindiennes) et de sources écrites, trouvées principalement dans les archives de l'église de la communauté anicinabe de Pikogan, où la majorité des études sur le terrain ont été effectuées. Une des difficultés de ces archives résidant dans le fait qu'elles ne sont pas systématiquement indexées ${ }^{6}$, faute de moyens. Les faits retenus ont été confirmés oralement par des personnes se souvenant des événements pour y avoir participé. Enfin, des archives visuelles privées (de familles anicinabek) m'ont été prêtées à des fins de consultation. Bien qu'ayant pris en compte, dans la récolte des données, les points de vue de prêtres catholiques, c'est bien plus au point de vue algonquin que j'ai voulu prêter attention. Mais regarder les deux simultanément m'a permis d'entrevoir les moments où le dialogue avait pu être entrecoupé de quiproquos. Ainsi, il est apparu que les Anicinabek, ayant en apparence totalement intégré les pratiques de dévotion et le calendrier de cérémonies religieuses promus depuis la deuxième moitié du XIX ${ }^{\mathrm{e}}$ siècle par leurs missionnaires, y ont également investi et théâtralisé un imaginaire social et cosmologique en transformation.

La période retenue pour cet article est celle comprise entre les années 1950 et les années 2000. Plusieurs raisons ont présidé à ce choix: la fin des années 1950 a marqué un tournant dans l'histoire des Anicinabek, de ceux de l'Abitibi dont je vais spécifiquement parler, mais aussi des autres

4. Ce projet est financé par une subvention d'équipe du Fonds québécois de recherche sur la société et la culture (FQRSC) sur le thème des «dynamiques religieuses autochtones des Amériques ». L'équipe est dirigée par Robert Crépeau, professeur d'anthropologie à l'Université de Montréal.

5. Je précise que les informations dont il est question dans cet article ont été recueillies auprès de personnes nées avant 1964. Les entrevues dont je me suis servie ont été effectuées entre 1996 et 2009.

6. Les rapports et lettres, trouvés dans le sous-sol de l'église de Pikogan, ne sont pas toujours datés et signés. 
régions. À cette époque, comme je l'ai dit plus haut, ils sont déjà tous catholiques et, du moins les aînés le racontent-ils, ils ont confiance en leurs missionnaires. Depuis le XIX ${ }^{e}$ siècle, ils sont affectés par le problème de l'alcoolisme (Bousquet 2005) et ils ont vécu de terribles épidémies et des famines qui les ont décimés. Leur économie a sombré avec la chute du prix des fourrures et avec la colonisation des régions où ils vivent. Ils sont de plus en plus marginalisés, car ils ne peuvent plus vivre à l'année sur leurs territoires de chasse, obligés de trouver des revenus d'appoint, et ils n'ont nulle part où aller. Ils reçoivent des rations des agents des Affaires indiennes, dont ils dépendent pour trouver du travail. Ils acceptent d'envoyer leurs enfants dans des pensionnats indiens (ils n'ont de toute façon pas le choix), en particulier celui de Saint-Marc-de-Figuery (Amos), dirigé par les Oblats de Marie Immaculée. Enfin, ils vivent dans une province où le clergé est tout-puissant et règle la vie, la morale et la politique de toute la société. À partir des années 1950, les Anicinabek deviennent sédentaires dans des villages bâtis sur le modèle de la paroisse québécoise, avec l'église sur la rue principale (Bousquet et Morissette 2008). Même s'ils ne désirent pas vraiment y vivre, la réserve représente pour eux, à défaut d'un autre choix, la promesse d'un meilleur accès au nouveau marché du travail, de la fin des menaces de disette avec la protection du gouvernement et de la proximité des soins de santé. Mais tout ce contexte va changer rapidement : contrairement à ce qu'on avait fait miroiter à leurs parents, les enfants qui sortent des pensionnats ne parviennent pas à s'insérer dans l'économie dominante. Les problèmes sociaux, liés à l'alcoolisme, ne vont faire qu'empirer, avec l'oisiveté liée au chômage et l'argent de l'aide sociale. De nouveaux problèmes de santé émergent (diabète, obésité) et la Révolution tranquille ainsi que les abus d'autorité de la part du clergé remettent en question le pouvoir de celui-ci. En outre, l'organisation sociale des Anicinabek, qui auparavant passaient dix mois de l'année avec leur seule famille et seulement un ou deux mois avec le reste des familles de leur bande, doit s'adapter à la promiscuité continuelle de la vie dans un village, ce qui affecte leur sens de la communauté, qui n'était pas fondé sur le voisinage (Bousquet 2009). Quand, dans les années 1980-1990, les anciens pensionnaires racontent au grand jour les traumatismes vécus dans les pensionnats et quand deux prêtres de paroisses amérindiennes sont

7. Chez les Anicinabek, le sens de la communauté était fondé sur la parenté, les alliances politiques, les relations économiques (partenariats de chasse par exemple), mais pas sur le vivre ensemble, qui n'avait lieu que l'été. 
reconnus coupables de pédophilie, la crise de confiance est totale. On peut donc dire que la période située entre les années 1950 et les années 2000 correspond à des bouleversements majeurs, venant s'ajouter aux changements sociaux les ayant précédés, devant lesquels les Anicinabek doivent trouver des réajustements dans tous les domaines, y compris leur échelle de valeurs et leur morale.

Je tenterai donc de démontrer que l'étude des rituels catholiques permet une lecture, dans le temps, de l'éthique anicinabe, en fonction des éléments qui ont modifié le mode de vie et de leurs conséquences. Pour ce faire, je commencerai par replacer ces rituels dans la culture catholique québécoise et dans les relations des Amérindiens avec les Eurocanadiens avant le passage à la sédentarité. Puis j'aborderai les transformations de l'ordre sociocosmique anicinabe et ce que les rituels révèlent de l'éthique anicinabe, depuis la sédentarisation, et j'insisterai sur le caractère pragmatique de sa philosophie sous-jacente. J'étudierai deux rituels en particulier: la bénédiction du matériel de chasse et les fêtes de Kateri. Enfin, alors que la pratique des rites catholiques a presque disparu chez les Anicinabek, je regarderai si d'autres rites collectifs sont venus les remplacer ou par quels autres biais on peut aborder cette éthique de nos jours. Cet article n'a pas de prétention théorique, mais vise plutôt à proposer diverses pistes analytiques sur un sujet encore peu documenté.

Avant de commencer, il faut rappeler que les missionnaires du $\mathrm{XIX}^{\mathrm{e}}$ siècle, et probablement un grand nombre de ceux qui leur ont succédé, jugeaient, comme la plupart de leurs contemporains dans la société civile jusque dans les années 1960 et 1970, que les Amérindiens étaient sauvages, pauvres, ignorants et qu'ils se conduisaient comme des enfants. C'est pourquoi leurs points de vue sont souvent exprimés en des termes très paternalistes, qui étaient la norme au moment où ils écrivaient. Les points de vue anicinabek, eux, résident dans les récits oraux qui se transmettent dans les communautés et qui sont tributaires (comme les récits écrits par ailleurs) de la perception de l'émetteur et du destinataire, ici des personnes dont les opinions ont évolué, au cours de leur vie et en fonction de l'époque.

\section{1. «Ils se sont surpassés!»}

C'est un événement que la visite de l'évêque! Tous tiennent à le recevoir de leur mieux $[\ldots]$ Je les laisse libres de préparer à leur guise décorations, chants et réception... Ils se sont surpassés! Sur le chemin qui conduit du 
quai à la chapelle, se dresse un magnifique arc de triomphe. Le parcours du cortège est bordé d'arbres enguirlandés et de drapeaux variés. (Martel 1934)

Quand, en 1934, le père Martel décrit la visite de Mgr Rhéaume au lac Abitibi pour procéder à des confirmations pendant la mission d'été, le cérémonial de ce genre d'événement est implanté depuis les années 1840. Son déroulement suit toujours la même trame: les Amérindiens préparent, avec des branches d'arbres et des rubans, des décorations pour baliser le chemin qui va conduire au lieu de célébration, lui-même également dressé avec tout le faste possible, autour d'une estrade et d'un autel. Ils tirent des coups de fusil dès qu'ils aperçoivent les canots escortant l'évêque, qui bénit la foule à son arrivée. Puis, en cortège, portant bannières et drapeaux, les Amérindiens escortent l'évêque, en chantant des cantiques, jusqu'au lieu où celui-ci dit la messe, devant tous les fidèles agenouillés. Enfin, l'évêque et les prêtres présents sont invités à partager un grand repas communautaire ouvert par un discours de bienvenue du chef et l'évêque distribue des cadeaux (médailles, dragées, images). Le même cérémonial est appliqué à toutes les célébrations impliquant des processions (fêtes du SaintSacrement, de la Sainte Vierge, etc.): au goût des missionnaires, les cortèges sont plus ou moins ordonnés et les décorations plus ou moins élaborées, mais les Amérindiens ont appris à marcher selon l'ordre prescrit par les cérémoniaires de l'Église catholique, à préparer les objets et les lieux nécessaires, à réciter et à chanter les répons et les hymnes et à procéder à la gestuelle appropriée ${ }^{8}$.

Pour comprendre ce que représentait ce type d'événements pour les Anicinabek, il faut d'abord pouvoir replacer les rituels catholiques dans leur contexte général: au Québec depuis le XIX siècle, puis dans le contexte particulier de l'évangélisation des Amérindiens, enfin dans les relations de ces derniers avec les autorités eurocanadiennes. Tout d'abord, au XIX ${ }^{e}$ siècle, le Québec a été le théâtre d'une reconquête catholique, comme l'explique René Hardy (2007). Après avoir perdu une grande partie de son influence, l'Église catholique, avec le soutien de l'État, va regagner son autorité après 1837 , au point de vue politique comme au point de vue moral. À partir des années 1820-1830, le clergé va s'employer à développer les pratiques de dévotion et, surtout, en les organisant tour à tour tout

8. Pour des descriptions de ces cérémonials chez les peuples algonquiens du Québec, voir dans les Annales de la propagation de la foi: Arnaud (1877, 25-27), Proulx (1882, 119-120: Témiscamingue, août 1881), Proulx (1892, 231-233 à Wemontaching et 240-241). 
au long de l'année: mois de saint-joseph (mars), culte à la Vierge au mois de mai, exercice des Quarante Heures à la Toussaint (culte au Saint Sacrement), exercices du Rosaire, dévotions au chemin de croix, les rituels (qu'ils soient domestiques ou dans l'espace public) étaient conçus de manière à occuper tout le calendrier (Remiggi et Rousseau 1998; Hardy 2007). Quant à la Fête-Dieu, qui avait lieu soixante jours après Pâques, avec ses processions et ses reposoirs, elle devint un sommet de l'année religieuse. L'adoucissement des règles d'accès à la communion et à la confession, la propagation des sociétés de tempérance et la multiplication des associations de piété (Hardy 2007) furent d'autres exemples de changements de stratégie du clergé pour «inculquer cette nouvelle culture religieuse » (77):

Plus exubérante et démonstrative, faite de dévotions multiples, de fréquentes génuflexions, de signes de croix et de nombreuses processions et manifestations sur la place publique, la piété dite "ultramontaine» se manifeste par des signes tangibles, mais elle est également faite d'une volonté d'imposer le respect intégral des prescriptions du jeûne et de l'abstinence, de la messe dominicale, de la communion et de la confession annuelles. (Hardy 2007, 77)

Les Anicinabek, comme les autres peuples algonquiens au nord du Saint-Laurent ${ }^{9}$, avaient, à l'orée du XIX ${ }^{\mathrm{e}}$ siècle, déjà eu des contacts, continus dans le temps mais irréguliers, avec des missionnaires. Après le tracé de la route des missions jusqu'à la baie d'Hudson par l'abbé de Bellefeuille, à partir de 1836, les missions «du Nord» furent confiées aux Oblats en 1844 (Riopel ca 1991). Par la suite, les Oblats firent chaque année le tour des groupes anicinabek pendant les rassemblements estivaux dans les postes de la Compagnie de la Baie d'Hudson, sites où ils construisirent des chapelles, baptisèrent les mourants, enseignèrent le catéchisme et s’informèrent de la consommation d'alcool des Amérindiens. Ils cherchèrent également à identifier des Métis $^{10}$ ou autres personnes, à la fois catholiques et parlant la langue vernaculaire, susceptibles de pouvoir instruire les Amérindiens en leur absence. Pendant la mission, destinée à catéchiser et

9. Les Amérindiens du Québec n’ont pas tous été évangélisés au même moment. Les Premières Nations proches des grands centres le long du Saint-Laurent, comme les Mohawks, les Hurons-Wendat, les Mi'kmaq ou les Abénakis, étaient déjà largement empreintes de culture catholique au XIX ${ }^{\mathrm{e}}$ siècle.

10. Le terme «Métis » était utilisé par les missionnaires pour désigner les descendants de mariages entre des Amérindiens et des Blancs. 
à dispenser les sacrements, les missionnaires faisaient faire des «exercices » à leurs nouveaux fidèles, comme des récitations de prières et l'assistance à la messe. Dans la lignée de la reconquête qui était lancée auprès des Québécois, ils organisèrent des événements spectaculaires et des pratiques de dévotion, dont les Quarante Heures (Proulx 1882), les cultes mariaux, la Fête-Dieu, etc. Les Anicinabek, d'abord réticents puis paraissant se convertir, incorporèrent la gestuelle du catholicisme ultramontain, comme s'agenouiller, se signer, marcher en chantant dans un ordre prescrit. Le succès de ces manifestations semble avoir été tel que certains missionnaires interdirent parfois de faire des processions comme mesure de rétorsion afin de contraindre leurs ouailles à s'amender. Ainsi, le père Gougeon, à la mission de Wemontaching (Atikamekw), voulait punir un excès de boissons alcooliques:

Je ne vous parlerai point de procession cette année. J'ai été assez mécontent de mon monde que je leur ai dit qu'ils ne méritaient pas d'avoir des processions. Corrigez-vous d'abord et ensuite vous aurez droit de faire une démonstration publique de votre foi et de votre piété. (Gougeon 1880, 226)

Selon les missionnaires, les Anicinabek reconnurent la légitimité de ces châtiments et adhérèrent tellement à ces pratiques qu'ils demandèrent aux missionnaires de venir s'installer de façon permanente parmi eux. L'attitude des Anicinabek de l'époque semble si soumise qu'elle en est presque incompréhensible, compte tenu du fait que leur fonctionnement social était fondé sur l'idée d'égalité entre les humains. Leurs descendants qui ont connu les pensionnats indiens ou qui sont des générations suivantes sont d'ailleurs convaincus que leurs ancêtres se sont fait manipuler mentalement et qu'ils ont subi des lavages de cerveau. Ne voulant pas rentrer dans ce débat qui leur appartient, je pense qu'il faut revenir à une hypothèse déjà proposée par les ethnohistoriens, à savoir que l'acceptation d'une nouvelle religion ne signifiait nullement le rejet des croyances traditionnelles, mais permettait peut-être, au contraire, d'améliorer les relations avec le monde des esprits (Viau 1995, 151): pour Riopel (2003), les Anicinabek "voient davantage dans la cérémonie du baptême un rituel visant à affermir leur alliance avec les chrétiens que l'adoption d'une nouvelle religion». "Nos ancêtres n'étaient pas fous ", "ils ont pensé que ce serait bon pour nous ", disent les aînés, qui peuvent être virulents contre les missionnaires en regardant l'histoire avec la distance de l'expérience, mais qui n'ont pas pour autant abandonné le christianisme. Si on engage la conversation sur le sujet de l'adhésion au catholicisme, les informateurs âgés lient, dans 
leurs discours, l'adoption d'une nouvelle religion avec l'apport de vêtements, de nourriture, d'outils, à une époque où les Anicinabek connaissaient la misère due à la baisse des ressources fauniques (gibier qui fournissait, rappelons-le, les vêtements, les outils, la nourriture, etc.). Si l'on peut y voir ce que Gélinas (2003) appelle des «manifestations d'esprit pratique» dans un article sur les Atikamekw, les aînés y ajoutent une dimension spirituelle: "pour avoir de la force ${ }^{11}$ » (mackawisiwin, être fort). Dans la cosmologie anicinabe, l'univers visible côtoie l'univers invisible et les bonnes relations avec l'un se répercutent dans l'autre.

Quand on regarde de plus près les caractéristiques de ces rituels lors de leur introduction en milieu amérindien, l'idée d'alliance apparaît d'autant plus pertinente ${ }^{12}$. En particulier, prêtons attention aux éléments suivants, qui ne font plus vraiment de sens pour les Anicinabek actuels à part qu'ils font partie de la tradition, de la "bonne manière de vivre", ce qui en fait des objets empreints de force: les décorations avec des rubans (ou ce qui en tient lieu, comme des mouchoirs), les décorations avec les branches d'arbre et les coups de fusil. Bien que ne faisant pas partie des rituels catholiques en tant que tels, j'inclus aussi les repas communautaires. Tout d'abord, les rubans, notamment de soie, semblent avoir été adoptés par les Amérindiens à partir du xviII ${ }^{\mathrm{e}}$ siècle dans l'Est canadien (Pannabecker 1996). Pour eux, la difficulté de s'en procurer en fit un objet de prix. Au XIX ${ }^{\mathrm{e}}$ siècle, le ruban devint un cadeau diplomatique typique du gouvernement. Par exemple, dans la région des Grands Lacs, «historical documents clearly indicate that European-produced ribbon was available to Great Lakes Indians through trade and as diplomatic gifts » (Pannabecker 1996, 268). L'importance cérémonielle des rubans se retrouve en contexte chamanique, où ils faisaient partie des offrandes faites à l'ours (animal le plus respecté de la cosmologie anicinabe), avec le tabac, les breloques de métal et les morceaux de tissu (Hallowell 1926).

11. Le mot «force», choisi par ma traductrice anicinabe, veut rendre différentes idées que les entrevues avec les aînés ont énoncées et qui sont liées entre elles: le savoir, l'intelligence et la lucidité (nisit), et le pouvoir, au sens de pouvoir spirituel. Ridington (1988) a d'ailleurs montré l'association, chez les peuples autochtones du subarctique, du savoir, du pouvoir et de l'autonomie individuelle.

12. Notons d'ailleurs que Delâge $(1991,55)$ en avait avancé l'idée pour les XVII et XVIII ${ }^{\mathrm{e}}$ siècles: «En s'alliant avec les Français, les Amérindiens concevaient qu'il en irait des connaissances religieuses comme des autres domaines touchés par l'alliance (économique, militaire, etc.): chacun échangerait et s'initierait dans la tradition de l'allié». 
Les décorations de branchages et de feuillages, elles, pourraient aussi bien avoir été introduites par les Jésuites qu'avoir été dès le départ autochtones (Clair 2006, 85-86). Quoi qu'il en soit, elles formèrent, dès le $\mathrm{XVII}^{\mathrm{e}}$ siècle, l'ornementation des lieux préparés par les Amérindiens lorsqu'ils recevaient la visite d'un évêque et la toile de fond des crèches, des spectacles de chants, etc. (Clair 2006, 85-86) ${ }^{13}$. Elles faisaient également partie de l'élaboration de rituels diplomatiques comme le cérémonial de condoléances (Savard 1996, 140) et il semble évident, de par la nature des relations des Anicinabek avec leur territoire (qui était à la fois leur maison, leur moyen de subsistance, leur magasin, un lieu empreint de sacré, etc.), que l'usage de végétaux dans le contexte catholique pouvait être perçu par les Anicinabek aussi bien comme étant chamanique que comme manière de théâtraliser le politique. Enfin, la forêt représentait toute la richesse des Anicinabek, d'où l'incompréhension que l'on perçoit chez les aînés actuels quand ils évoquent le fait que les Blancs les trouvaient pauvres à l'époque du semi-nomadisme. Le père Lacasse (1881), en 1880, relate un fait similaire lors des préparatifs de la visite d'un évêque à Pointe-Bleue:

Ils n'avaient à leur disposition que les arbres et les feuilles de la forêt, plus deux ou trois châles et trois ou quatre mouchoirs que les jeunes gens de la tribu voulaient utiliser sous forme de pavillons. Je vous concède bien volontiers, cher ami, que je n'ai pas le génie de l'invention, mais j'avais beau réfléchir, je ne voyais pas la possibilité, avec de tels moyens, de faire quelque chose de passable. Rencontrant un de mes Sauvages, je lui fis part de ma crainte; il parut fort surpris de mon aveu: «Nous ne sommes pas si pauvres, dit-il, puisque nous avons toute la forêt à notre disposition. Il y en a, va, de l'épinette et du sapin dans le bois».

Les coups de fusil, eux, ou l'explosion de pétards proviennent de traditions populaires catholiques, en Europe. Ils accompagnaient particulièrement les processions de la Fête-Dieu. Cette tradition se retrouve également en Nouvelle-France: citant le Journal des Jésuites relatant la Fête-Dieu de mai 1646, Gallat-Morin $(2001,49)$ rapporte que «la procession s'ébranle au son de la cloche de la paroisse, de coups de canon et de salves répétées de mousquets et de fusils ». Dans un contexte diplomatique, tirés pour chasser «le mauvais air de la guerre» (Savard 1996, 208) ou pour saluer l'arrivée d'ambassadeurs importants, les coups de fusil font partie de la pompe d'un protocole connu de tous les Amérindiens depuis

13. Cuoq $(1893,141)$ précise que le nom donné par les missionnaires à la Fête-Dieu est Anibican patakising, "quand on plante du feuillage". 
au moins le XviI ${ }^{\mathrm{e}}$ siècle. Depuis le début du $\mathrm{xx}^{\mathrm{e}}$ siècle ou peut-être de la fin du XIX ${ }^{\mathrm{e}}$, tirer des coups de fusil en l'air fait aussi partie des célébrations du jour de l'An. Dans la perception anicinabe, il est raisonnable de penser que les coups de fusil évoquent également le bruit du tonnerre, inimiki, qui fait partie des personnes autres qu'humaines ${ }^{14}$ que les Anicinabek respectent et avec lesquelles ils négocient leur place dans le monde.

Enfin, les repas communautaires ou makocanak (prononcer makouchanak) étaient et sont toujours très importants dans la sociabilité anicinabe. Chez les Anicinabek, comme chez les autres Algonquiens, ils étaient offerts lors de visites de personnages importants, mais aussi lors des funérailles (Laniel 1893), ainsi que lors des départs et des retours de la chasse, pour se restaurer et pour se concilier les esprits des animaux, qui font partie de leur univers social. De nos jours encore, les makocanak marquent les retours de chasse, les funérailles, les visites de personnes que l'on veut honorer, ainsi que le jour de l'An et d'autres célébrations ponctuelles. Leur tenue signale, pour les informateurs, que la communauté «va bien» et que les gens y sont «fidèles à ce qu'ils sont". En bref, tous ces éléments du rituel accompagnaient aussi bien les alliances militaires que les alliances religieuses que les Amérindiens scellaient avec les Blancs.

Rappelons que les missionnaires lancèrent un mouvement soutenu d'évangélisation dans un contexte de famines, d'épidémies et de bouleversements de l'univers des Anicinabek. Comme nous le verrons plus loin, les Anicinabek conçoivent les événements dans un système de pensée pragmatique, où n'est vrai que ce qui fonctionne réellement et où la connaissance se révèle par l'expérience. Ainsi, leur acceptation des processions et autres célébrations, si elle a été aussi inconditionnelle que les chroniques missionnaires semblent l'affirmer, est sans doute plus compréhensible si l'on pense que les missionnaires avec lesquels ils faisaient ainsi alliance leur apportaient des moyens spirituels et matériels pour lutter contre l'envahissement des territoires par les chasseurs blancs et par les chantiers forestiers (grâce à leur influence auprès du gouvernement), contre l'abus d'alcool (par les mouvements de tempérance), contre la famine (grâce à des vivres), etc. Les ritualisations de ces alliances théâtralisaient la recherche de leur efficacité, pour communiquer avec les esprits catholiques et en obtenir les faveurs.

14. La cosmologie algonquine, de type animiste, est fondée sur l'idée que l'univers social n'est pas composé que d'humains, mais aussi d'autres «personnes » avec lesquelles il faut socialiser et qui ne sont pas humaines, comme les esprits des animaux, les vents, le tonnerre, les astres, etc. 
C'est ce que suggère un passage des mémoires du père Fortin, curé chez les Innus, dont le contenu concorde avec ce que j'ai entendu chez les aînés anicinabek de Pikogan:

En effet, à Mingan vers les années 1963, 1964, 1965, j'avais négligé d'organiser la procession de la Sainte Vierge au cours de ces étés. Or, un jour d'automne, le bon vieux Damien m'arrive et me dit: «Ça va mal dans le village, Père! C'est parce que tu ne fais plus la procession de la Sainte Vierge, Sainte-Marie ne s'occupe plus de nous! (Fortin 1992, 12)

Je souligne ici, parce que j'y reviendrai plus loin, que le missionnaire se fait rappeler ses devoirs, ce qu'il interprète comme une marque de dévotion à la Sainte Vierge, mais qui pourrait aussi être interprété comme un rappel, par son interlocuteur, de sa fonction et de sa place chez le peuple qui l'accueille.

\section{Comment mener une bonne vie}

Au début, le temps de la mission se résume à quelques semaines, voire à quelques jours dans l'année. Le calendrier catholique est donc concentré sur une courte période, même si les missionnaires prescrivent des exercices quotidiens à accomplir en leur absence. Puis le passage à la sédentarité rend possible l'installation permanente des missionnaires au milieu de leurs ouailles. Les célébrations prennent alors place dans l'enceinte des villages amérindiens, où l'église est toujours un des premiers bâtiments à être construits (Bousquet et Morissette 2008). Avec le changement de mode de vie, les différents espaces de l'univers des Anicinabek n'ont plus la même signification qu'auparavant: si le milieu urbain va être un des derniers à être investi, le village devient le premier lieu d'habitation et la forêt, lieu des ancêtres et des animaux, va alors être un espace de plus en plus lointain dans les pratiques quotidiennes. Les Anicinabek n'abandonnent pas leurs territoires familiaux pour autant: dans les années 1950 et 1960, les camps de chasse sont occupés dès que les travaux saisonniers sont finis. Puis, au fil des décennies, ils y font des séjours plus espacés, à cause du travail et de l'école des enfants. Mais les semaines de piégeage, de pêche et de chasse, qui ont lieu au printemps, à l'été et à l'automne, sont encore bien suivies de nos jours. Ces changements de signification de l'espace et le bouleversement du mode de vie imposent une nouvelle lecture des rituels qui s'y déroulent. Je m’attarderai ici à deux rituels en particulier: les bénédictions de matériel de chasse et les fêtes de Kateri. Je les ai choisis parce qu'ils 
avaient lieu ponctuellement et parce qu'ils sont les plus fréquemment cités par les informateurs de Pikogan quand ceux-ci évoquent les moments heureux avec les missionnaires (l'évangélisation du XIX ${ }^{\mathrm{e}}$ siècle n'étant pas considérée, elle, comme une période de bonne entente avec les «Robes noires»). J'émets l'hypothèse que les significations de leurs performances reflètent les transformations de la sociabilité anicinabe et des relations de pouvoir avec le clergé et avec les autorités, ainsi que les transformations de l'ordre cosmique. Je partirai également de l'idée, dans la lignée des recherches de Gagnon chez les Innus (2005, 140-141), que ces fêtes font partie, pour les Anicinabek, d'un système religieux qui résulte d'une interpénétration de divers éléments du catholicisme et du chamanisme.

Pour comprendre les performances de ces rituels, il faut d'abord savoir pourquoi les gens les faisaient ou y participaient. La première raison invoquée par les Anicinabek est l'obtention de faveurs, qui pouvaient aller d'une chasse fructueuse à une météo favorable, en passant par la guérison de quelqu'un, la cessation de fléaux sociaux (notamment dus à l'abus d'alcool), etc. Une autre raison est l'accomplissement d'un rêve. Une autre enfin est le devoir envers la collectivité, l'importance de se réunir pour être plus forts et pour garantir l'ordre moral et social général. Ensuite, il s'agit de saisir la portée de ces raisons, en les replaçant dans la philosophie anicinabe sur la manière de mener «une bonne vie» (pimatisiwin ${ }^{15}$ ) en respectant l'éthique anicinabe. Comment, en fait, agir au mieux, en tant qu'être humain, vis-à-vis de tout ce qui nous entoure? L'éthique anicinabe est fondée sur deux principes a priori antinomiques (ou du moins vus comme tels dans la pensée occidentale): le sens de l'individualité, qui commande que l'individu se prenne en charge lui-même et n'ait pas à subordonner ses aspirations à celles de sa collectivité, et le sens de la communauté, qui implique des devoirs envers cette même collectivité, devoirs qui sont inclus dans une idéologie de réciprocité. Cette réciprocité vaut d'ailleurs également pour les êtres autres qu'humains et pour tout ce qui compose le territoire: si on préserve le territoire, le territoire rendra quelque chose en échange. Les Anicinabek ne sont ni strictement individualistes ni strictement communautaristes: ils sont les deux. Ils reconnaissent qu'il est impossible de faire certaines choses individuellement, que ces choses doivent donc être faites en groupe. Les intérêts personnels ne priment pas sur l'intérêt collectif et vice versa. Mais si un individu, par égoïsme, met en danger un aspect de la vie collective, il s'expose à subir des sanctions, car

15. Sur la notion de pimatisiwin, voir Bousquet (2012b). 
il touche alors aux intérêts personnels des autres. Or, la non-ingérence dans la vie des autres est un principe anicinabe fondamental.

Mais comment savoir si l'on a agi au mieux? Seule l'expérience va permettre de le savoir et, particulièrement, les conséquences de l'action, dont il faut tirer des leçons. La philosophie anicinabe n'est pas strictement pragmatique, car les effets d'une action ne sont pas identifiés à l'avance. Mais elle est ancrée dans le concret: pour pouvoir donner son point de vue sur une idée, il faut l'avoir expérimentée ou en avoir vu les implications pratiques. Plusieurs auteurs ont évoqué le pragmatisme et l'empirisme (philosophie qui place l'expérience à l'origine de toute connaissance valide) des populations algonquiennes, notamment face aux entreprises d'évangélisation. Ainsi, Conkling $(1974,21)$, au sujet des Wabanakis du XvII ${ }^{\mathrm{e}}$ et $\mathrm{XVIII}^{\mathrm{e}}$ siècles, explique que: "the Indians could see that some ideas were no longer very useful in the manipulation of reality. The pragmatism and empiricism of the Indians [...] is representative of the broad pattern of religious testing at this time ". Conkling va en fait jusqu'à comparer la conversion des Wabanakis au catholicisme à l'adoption, par un scientifique, d'un nouveau paradigme. Il avance en effet que:

It is argued [...] that the test of religious beliefs, not just the authority of religious persons, was also pragmatic in part and that, indeed, there were tests of these beliefs just as there are tests of scientific ideas in the laboratory. The choice of which ideas to believe in, like the choice of which leaders to obey, Indian or French, was made on the basis of considerations of economic benefits and efficacy of political organization, of course, but also by the degree to which dreams, oracles, magic charms, etc., permitted the successful manipulation of reality. The process of religious conversion, which sometimes seems irrational, was no more divorced from ideas of explanatory and predictive usefulness than the conversion of a physicist from one scientific paradigm to another. (Conkling 1974, 2)

La même idée ressort dans des textes successifs de Kenneth Morrison $(1986 ; 1990)$ sur l'évangélisation des Montagnais au XviI ${ }^{\mathrm{e}}$ siècle: "The priest did not comprehend that what mattered to the Montagnais was concrete experience, and he did not understand that rituals attached to hunting ensured proper relations with these mythological beings. The Montagnais' religious discrimination was everyday and practical» (1986, 6). C'est par ce pragmatisme et cette nécessité d'expérimenter et d'obtenir des preuves que Morrison interprète l'adoption par les Montagnais de nouveaux symboles, de nouveaux rituels et de nouvelles dévotions (à Jésus par exemple) «in order to deal with real and novel problems» $(1990,433)$. 
Cette attitude face à la vie a continué à être incomprise par le clergé du $\mathrm{xx}^{\mathrm{e}}$ siècle, ainsi que par les autres Eurocanadiens d'ailleurs:

La psychologie de ces Asiatiques, primitifs et ignorants, préfère ici de beaucoup les sacramentaux, les processions, les fêtes au cimetière. Les abstractions et même les sacrements ne sont pas leur fort. (S.-A. La Rochelle, o.m.i, assistant général et visiteur, Actes de Visite Générale de la Résidence NotreDame-du-Rosaire, Maniwaki, 16-17 juillet 1964, p. 55, Archives Deschâtelets)

Aborder l'éthique anicinabe, et algonquienne en général peut-être, sous l'angle des méthodes philosophiques que sont le pragmatisme et l'empirisme gagnerait à être encore approfondi. Il est particulièrement frappant qu'on puisse identifier des similitudes entre les acteurs amérindiens des Relations des Jésuites et les Anicinabek d'aujourd'hui, alors que plus de trois siècles les séparent. Outre cette continuité (apparente au moins), nous retiendrons que les connaissances ainsi dégagées impliquent, pour les Anicinabek, d'en tirer des conséquences, soit déterminer les meilleures actions à accomplir pour son propre bien-être ou pour le bien-être général. On pourrait dire que l'éthique anicinabe est de type conséquentialiste, puisque le conséquentialisme «nous prescrit d'accomplir en toutes circonstances l'acte qui, parmi toutes les lignes de conduite qui nous sont disponibles, aura les meilleures conséquences» (Stroud 2001, 152). L'analyse morale fondée sur l'évaluation des conséquences de ces actes permet de savoir s'il faut ou non les réitérer.

\subsection{Les bénédictions de matériel de chasse}

Venons-en maintenant aux descriptions des deux rituels, tels qu'ils m'ont été décrits à Pikogan surtout, mais aussi à Lac-Simon, en commençant par les bénédictions du matériel de chasse. Ces bénédictions, qui avaient lieu avant les départs à la chasse d'automne, c'est-à-dire à des dates qui n'étaient pas fixes, se déroulaient généralement un dimanche après la messe du matin. Pendant la messe, "un aîné prenait la parole [...] évoquant la chasse à venir, le territoire à exploiter ainsi que les prescriptions à suivre» (Bousquet et Morissette 2008, 15). Chaque groupe de chasse familial (souvent correspondant à une maisonnée) disposait l'ensemble des bagages devant sa maison: sacs de vêtements, pièges, fusils et munitions, chandelles, canot, haches, scies, provisions (farine, poudre à lever, sel, sucre, thé). Souvent, le canot et les fusils étaient décorés de rubans. Le 
prêtre passait devant chaque maison pour bénir tous les objets, auxquels les gens pouvaient ajouter des images pieuses, des chapelets et des croix, à accrocher dans le camp de chasse. Le prêtre bénissait aussi de l'eau qui, apportée au camp, servait à protéger celui-ci. L'eau bénite rentrait également dans la préparation de médicaments et était utilisée pour frictionner les gens malades. Le makocan n'avait en général pas lieu ce jour-là, mais plutôt au retour de la chasse: après une messe d'action de grâces, toute la bande se réunissait pour manger ensemble le gibier et le poisson rapporté.

Les bénédictions de départs de chasse existaient aussi chez d'autres Algonquiens. Ainsi, elles avaient notamment lieu chez les Innus, selon un déroulement à peu près similaire, comme en témoigne le père Fortin:

Chez les Indiens, il y a tout un rituel à suivre avant le départ pour la chasse. D'abord, un bon dimanche après la messe, c'est la bénédiction des canots, neufs et vieux ou non finis, dehors à la porte de l'église. Ensuite, les gens apportent bocaux, cruches ou grands vases pour les remplir d'eau bénite. Chaque famille en apporte ainsi que des petits contenants de sel à être bénis. En plus, parmi les objets à bénir, il y a les fusils qui sont tous décorés d'un ruban de couleur, des chandelles de tentes, de la gomme de sapin, des images, des petits cadres, des médailles grosses, moyennes et petites, des chapelets, des crucifix, des petites croix. Ces dernières années, la grande chasse étant presque abandonnée, j'avais négligé et même abandonné ce rituel. Or, on vient de me rappeler mes oublis et maintenant, à l'automne, au début de septembre, on fait encore toutes ces bénédictions. (Fortin 1992, 103)

Le choix du dimanche pour effectuer le rituel paraît logique dans la perspective catholique. Il l'est aussi dans la perspective anicinabe: on ne chasse pas le dimanche, car cela apporte la malchance. Ce legs du catholicisme, selon lequel le dimanche est jour de repos, est encore respecté par un grand nombre de chasseurs, qui n'y voient pas du tout un héritage chrétien, mais une évidence issue de l'expérience: "Ce n'est pas bon de partir un dimanche. Il arrive toujours une bad luck quand on part le dimanche» (homme de 46 ans, Pikogan, 22 septembre 1996). Or, tout départ à la chasse nécessite de prendre en compte les facteurs métaphysiques comme les facteurs pragmatiques, prendre en compte les mouvements de la nature, la météo, les esprits, les humeurs humaines, afin de mettre toutes les chances de son côté. Une des fonctions principales du rituel était de protéger contre la malchance, par exemple: faire une mauvaise chasse, avoir un accident ou encore faire une mauvaise rencontre en 
forêt (animal ou esprit). En bref, les éléments du rituel rendaient les gens plus forts.

Si le rituel avait clairement des vertus propitiatoires, compatibles avec le substrat chamanique du système de croyances anicinabe, il avait aussi d'autres qualités. Il montrait la fonction assignée au clergé, dont le rôle était de demander l'intercession de Dieu ou, plutôt, de Jésus (les Anicinabek font surtout référence à Jezos quand ils parlent de l'esprit qui intervenait dans ces bénédictions). Le prêtre était le régulateur de l'ordre cosmique et les gens étaient prêts à lui rappeler ses devoirs s'il y faillait. Il n'était donc plus un personnage tout-puissant, comme les premiers missionnaires des récits oraux, qui faisaient parfois peur, mais quelqu'un d'inclus dans la sociabilité anicinabe avec un rôle social déterminé. Rappelons également que le rituel avait lieu dans le village, endroit que, dans les décennies de sa célébration (les années 1960, 1970 et 1980), les Anicinabek considéraient encore comme leur étant étranger. Le rituel permettait de faire la jonction entre la réserve et le territoire, que les Anicinabek regardaient toujours comme l'espace par excellence de leur mode de vie. Il montrait également la perpétuation des «bonnes manières de faire» les choses: les bonnes manières de chasser, nécessaires pour vivre une bonne vie, et les bonnes manières de se comporter envers le monde invisible. L'intervention d'un aîné pendant la messe, qui prenait la parole en tant que chasseur expérimenté et reconnu par ses pairs, est un autre indicateur de la place de chacun dans la cosmologie. Jusqu'à Vatican II, les laïcs ne pouvaient prendre la parole pendant les célébrations eucharistiques, ne tenant (et seulement des hommes) que des rôles subalternes. Même après Vatican II, les aînés, très attachés aux traditions, ne tenaient pas de rôles actifs lors des messes. Mais le fait qu'un aîné faisait un discours est révélateur. D’une part, le curé ne pouvait parler à sa place, car il n'était pas reconnu pour ses connaissances en matière de chasse. D'autre part, un aîné pouvait s'exprimer en se mettant sur un plan égalitaire avec le prêtre, chacun assumant ses compétences. Enfin, les animaux et le monde de la forêt avaient encore une place prépondérante dans la cosmologie et ce, malgré la sédentarisation.

Ce que les Anicinabek regrettent aujourd'hui, ce n'est pas le contenu du rituel: ceux qui pensent qu'une bénédiction est nécessaire peuvent toujours se procurer de l'eau bénite et s'en servir pour appeler eux-mêmes la protection de Jésus. Les intérêts personnels ne sont ainsi pas menacés. Ils regrettent surtout que la tradition ne soit pas perpétuée, comme un lien de plus coupé avec le territoire et avec les manières de faire d'autrefois, et ils regrettent que la sociabilité anicinabe ait beaucoup changé: les gens ne se 
rassemblent plus beaucoup pour effectuer des fêtes collectives, ce qui, selon eux, affaiblit le corps social. Dans les deux communautés où les informations ont été recueillies, le rituel a pris fin au début des années 1990, car le nouveau curé qui venait d'arriver dans les deux paroisses, et qui remplaçait donc deux personnes, n'entendait pas le perpétuer. Comme nous le verrons plus loin, il ne voulut pas non plus ajouter à sa liste de tâches la présidence du comité d'organisation des fêtes de Kateri, qui tombèrent en désuétude. L'un des deux curés, qui avait été affecté successivement à l'une et à l'autre communauté, partit en outre pour répondre à des accusations de méfaits et de pédophilie, ce qui brisa durablement la confiance envers les prêtres en général.

\subsection{Les fêtes de Kateri}

Les fêtes de Kateri ou journées de Kateri, quant à elles, semblent avoir seulement existé chez les Anicinabek. L'Église du Canada fête le 17 avril16 (jour de sa mort en 1680) cette bienheureuse mi-iroquoise, mi-anicinabe, béatifiée en 1980 par le pape Jean-Paul II et canonisée en 2012 par le pape Benoît xvi. Mais si elle fait l'objet de dévotion chez de nombreux peuples amérindiens, notamment à travers les cercles Kateri qui priaient pour sa canonisation (Rigal-Cellard 2005; 2006), je n'ai trouvé de trace de célébrations particulières, telles que celles que je vais décrire, que chez les Anicinabek. Je différencie en fait les fêtes de Kateri de la Kateri Conference (Rigal-Cellard 2006), qui a pour but de travailler à l'inculturation du catholicisme pour mieux l'adapter au milieu amérindien et qui organise des conférences annuelles, avec Grande Entrée (comme dans les pow-wows), messes et ateliers. Les fêtes de Kateri, elles, étaient organisées par des curés et par des Anicinabek pour des Anicinabek.

Les journées de Kateri auraient commencé dans les années 1970 et se seraient terminées au milieu des années 1990. Elles auraient donc existé pendant une vingtaine d'années. Elles duraient en moyenne trois jours et avaient lieu, semble-t-il, au début du mois d'août. Selon un missionnaire oblat, rencontré le 26 janvier 2007 à Lac-Simon, il s'agissait d'un genre de "pèlerinage intercommunautaire»: une communauté recevait les autres. Le site choisi pouvait être soit un village, comme Pikogan, Timiskaming ou Kitigan Zibi, soit un terrain de chasse familial, soit encore un ancien lieu de retrouvailles estivales, comme le site de Kitcisakik où se trouve

16. Dans le sanctoral américain, elle est fêtée le 14 juillet. 
l'église. Outre les discussions, échanges, jeux et réjouissances entre familles, avaient lieu des activités programmées à l'avance. Les gens arrivaient le vendredi, avec leurs tentes. Le soir de cette journée-là, on faisait un grand feu, dit «de l'amitié », autour duquel on buvait du thé. Le samedi était une journée de prières et de chants. Une jeune Anicinabe avait été choisie par la communauté d'accueil pour personnifier Kateri et quelques bénévoles reproduisaient, sous forme de "pageant", un épisode de la vie de Kateri. La journée était clôturée par une messe. Le dimanche, l'évêque arrivait, salué par des salves de fusils, pour célébrer l'eucharistie. Tout le monde partait alors en procession jusqu'au lieu de la célébration : l'évêque, tenant l'ostensoir, était abrité sous un dais porté par quatre hommes, précédé et suivi par des gens portant les bannières des paroisses anicinabek représentées et chantant des hymnes ${ }^{17}$. Puis la messe était célébrée en plein air sur un site spécialement préparé à cet effet. Généralement sur une estrade, l'autel pouvait être un canot ${ }^{18}$ ou une table, dressé devant une image (ou une statue) représentant Kateri Tekakwitha. Les décors variaient selon les années: tipi (réel ou stylisé), croix en bois de bouleau, décorations florales, feuillages, dessins d'enfants. Parfois, les reliques de Kateri de la paroisse locale étaient apportées sur le lieu de la messe. Après la messe, avait lieu un grand makocan pendant lequel les chefs présents remerciaient l'évêque et les organisateurs et organisatrices, les chasseurs et les cuisinières et tous les gens qui s'étaient déplacés, puis tout le monde retournait chez soi.

On retrouve dans les journées de Kateri des éléments dont j'ai déjà parlé: les décorations en feuillages, voire tout le décor catholique du rituel qui est indianisé (Bousquet et Morissette, 2008), les salves de fusils, les discours de remerciement des chefs, qui font partie des protocoles diplomatiques, et les makocanak, auxquels on pourrait associer le fait de boire du thé. C'est la boisson anicinabe par excellence qu'on offre, avec un morceau de bannique (pain amérindien), à tout visiteur de passage selon les règles de l'hospitalité. La visite de l'évêque, qui perpétue une tradition commencée au XIXe, et la reproduction d'un cérémoniaire catholique qui évoque fortement la Fête-Dieu (dais, procession, bannières) suggèrent ce que Fourchard (1999), pour les cérémonies catholiques à Ouagadougou

17. Ces informations sont tirées d'entretiens, mais aussi de consultations d'albums photo de familles anicinabek. Au fil des années, à partir des années 1980, le dais au-dessous duquel l'évêque se plaçait a disparu.

18. Le canot comme autel fait penser à la fête du saumon qui a commencé à Natashquan en 1974, à la demande du chef Antoine Malec et du mouvement AA (voir Fortin 1992, 90 et 111-112; Lamothe 1977). 
pendant la première moitié $\mathrm{du} \mathrm{xx}^{\mathrm{e}}$ siècle, appelle la mise en scène du cérémonial colonial, où un imaginaire social, dominé par la hiérarchie du clergé, était proposé aux fidèles de façon théâtralisée. Les parcours choisis pour les processions montraient quels espaces publics faisaient partie des «lieux de la catholicité » (Fourchard 1999, 152), c'est-à-dire l'ensemble des espaces anicinabek: pas seulement le territoire et les anciens lieux de rassemblement estivaux, mais aussi les villages sédentaires.

Le choix de Kateri comme personnage du calendrier chrétien à laquelle l'hommage était rendu doit être souligné : il s'agit de la seule catholique amérindienne de l'Amérique du Nord à avoir été canonisée. Les Oblats ont beaucoup œuvré, avec les Jésuites, à développer cette dévotion et ce, particulièrement, depuis les années 1940 (la cause de Kateri Tekakwitha fut introduite à Rome en 1943). Un article de Vie Indienne (Anonyme 1965), ancien journal oblat, souligne six points pour lesquels, selon les Oblats, il fallait absolument obtenir la béatification de Kateri:

1- Elle était laïque.

2- Elle n'était pas de race blanche, mais appartenait à la race amérindienne, du vaste groupe ethnique mongol.

3- Elle était pauvre, d'une pauvreté semblable à celle des trois-quarts du monde moderne.

4- Elle était analphabète comme le sont des millions d'hommes du $\mathrm{Xx}^{\mathrm{e}}$ siècle.

5- Elle est une des gloires de l'Église des missions: la seule, semble-t-il, qui, sans être martyre, a eu ses vertus officiellement reconnues héroïques par Rome.

6- Elle deviendrait la première Indienne authentique à monter sur les autels. Quelle consolation et quel exemple pour les millions d'Amérindiens et surtout pour nos milliers d'Indiens du Canada. (Anonyme 1965, 3)

Kateri devait donc servir de modèle auquel se référer pour devenir un ou une meilleure catholique anicinabe, un modèle de valeurs chrétiennes et de rupture avec l'ordre chamanique anicinabe, une marque de réussite de l'évangélisation et de l'implantation durable du catholicisme chez les Anicinabek. De nos jours, on trouve encore fréquemment des portraits d'elle dans les maisons anicinabek, portraits qui étaient distribués par les curés des paroisses amérindiennes sous forme d'affiches, de petites images, voire de macarons à porter sur ses vêtements. Un article de Hélène Hervet, "L'Abitibi en mocassins », publié le 18 mars 1999 dans le magazine inter- 
net $\mathrm{La} \mathrm{Vie}{ }^{19}$, relate un exemple de la récupération anicinabe du discours oblat, ici par une guide touristique anicinabe: selon cette dernière, Kateri est "gardienne de nos lacs, de nos forêts et de nos rivières en péril ». Protectrice des missions, elle devint protectrice, dans le discours anicinabe, du territoire et du bien-être de celui-ci, remettant à l'avant-plan, dans une perspective très amérindienne, la place essentielle de l'univers de la forêt dans la cosmologie ${ }^{20}$.

La performance d'un "pageant» est le dernier élément qui retient notre attention. Les "pageants » religieux sont de petites pièces de théâtre, organisées en tableaux, qui représentent des histoires de la Bible ou de saints, ou autres scènes de l'héritage catholique. Nés au Moyen Âge en Europe, ils avaient lieu lors des carnavals et des grandes célébrations saisonnières. Au Québec et ailleurs en Amérique du Nord, ils ont d'abord été utilisés par les Jésuites, puis par d'autres congrégations (dont les Oblats) pour évangéliser les Amérindiens et leur enseigner ces histoires. Walsh $(1994,19)$ qualifie ces «pageants » de "colonial religious drama ", " hibridization of medieval dramatic form and native ceremony». Dans le cas des journées de Kateri, les tableaux de la vie de la bienheureuse étaient joués par des Anicinabek afin que, par processus de mimèsis, ils puissent s'identifier à elle. Si Walsh n'explique pas ce qu'il entend par «native ceremony ", il fait cependant le parallèle avec les rituels collectifs hautement scénarisés des Amérindiens. On peut penser, notamment, à la tente tremblante chez les Anicinabek, rituel impressionnant où un chaman qualifié entrait pieds et poings liés dans une petite tente et la faisait trembler en y faisant venir des esprits animaux. Mais il reste que le contenu des «pageants» et leurs performances étaient contrôlés par le clergé, ce qui discrédite l'idée d'une cérémonie "native» (amérindienne).

Le déroulement des journées de Kateri rappelle donc bien plus les cérémonies inculquées au XIX ${ }^{\mathrm{e}}$ siècle que ne le fait le rituel de bénédiction avant la chasse. On y retrouve la mise en scène coloniale du leadership du clergé, ses stratégies d'enseignement de la culture religieuse catholique aux Amérindiens et la performance de l'alliance des Anicinabek avec les missionnaires. Les Anicinabek qui y ont participé, du moins ceux que j’ai

19. Voir <http://www.lavie.fr/archives/1999/03/18/1-abitibi-en-mocassins,2885003.php>, consulté en juin 2013.

20. Outre son rôle de protectrice des forêts et des rivières, Kateri Tekakwitha est réputée pour porter chance et pour protéger les voyageurs, tel saint Christophe, mais elle est également invoquée pour d'autres raisons, comme celle de venir en aide à un proche, par exemple (demander sa guérison morale et/ou physique, notamment). 
interrogés, n'ont oublié aucun de ces éléments. Ils déplorent la démonstration de la puissance des prêtres, mais, disent-ils à l'époque, ils les croyaient, ils les suivaient, ils pensaient que les curés avaient raison et qu'ils étaient des gens puissants, aussi bien au niveau séculier que sur le plan spirituel: n'étaient-ils pas des «envoyés de Dieu » ? En préparant bien ces fêtes et en y assistant, les Anicinabek pouvaient rechercher leur bienveillance. Les fêtes de Kateri étaient aussi des occasions de demander des faveurs à Jésus et à Kateri. Si les Anicinabek d'aujourd'hui ne regrettent pas du tout l'alliance avec les missionnaires (à part quelques-uns, dont une majorité d'aînés, qui restent convaincus), ils regrettent l'événement en lui-même qui faisait partie, selon eux, des démonstrations de leur fierté d'être Anicinabek. Les fêtes de Kateri évoquaient aussi les retrouvailles estivales, période de réjouissances de la vie traditionnelle, où étaient célébrés les mariages, renégociées les alliances entre chasseurs, où avaient lieu les grands rites collectifs et de nombreux jeux. Ce moment crucial du calendrier anicinabe et de son ordre social, qui a perdu son sens avec la sédentarité, était en quelque sorte «restauré » en un genre de fête nationale où étaient réactivés les fondements du sens communautaire: visites de la parenté, rencontres, échanges (de nourriture, de points de vue, d'histoires), rites collectifs.

Les journées de Kateri, créées par un Oblat qui fut prêtre au pensionnat indien de Saint-Marc-de-Figuery et curé de trois paroisses anicinabek, furent terminées le jour où ce même prêtre quitta sa dernière paroisse, les menottes aux poignets, au milieu des années 1990. Son successeur ne voulut pas reprendre la tête du comité d'organisation. De nos jours, il est toujours possible, par la prière, de chercher à s'attirer des dons de Jésus, de Kateri, ou d'autres membres du panthéon catholique. Mais l'identité anicinabe, même si elle a été influencée par l'empreinte du catholicisme, s'est beaucoup dissociée de la part catholique qu'elle avait incorporée. La question est alors de savoir si d'autres rituels peuvent remplir les rôles que les Anicinabek avaient assignés aux rituels catholiques et, surtout, si ces rôles ont encore un sens dans la conception contemporaine de l'ordre sociocosmique.

\section{Chamanisme, célébrations chrétiennes et panindianisme: un retour en boucle?}

Le procès, médiatisé, du prêtre pédophile, en 1996, fut suivi, quelques années plus tard (en 2002), par l'arrestation, pour les mêmes motifs, d'un autre père oblat, âgé de 69 ans, qui avait longtemps été curé de la commu- 
nauté atikamekw de Wemotaci. Déjà, depuis les années 1980, les anciens pensionnaires, devenus adultes, s'étaient mis à parler des sévices qu'ils avaient pu vivre dans leur enfance. En 1991, Phil Fontaine, chef national de l'Assemblée des Premières Nations au Canada, avait révélé publiquement avoir été abusé sexuellement quand il était lui-même dans un pensionnat. L'onde de choc provoquée par ces révélations, si elle eut des effets cathartiques dans les communautés, vida les églises anicinabek d'une grande partie de leurs fidèles. Elle eut aussi pour contrecoup et ce, bien qu'il soit difficile d'établir des liens directs de cause à effet, de permettre aux discours de remise en cause de la religion catholique et de ses représentants cléricaux de s'exprimer dans l'espace public anicinabe. Contrairement aux savoirs botaniques ou zoologiques ${ }^{21}$, par exemple, les savoirs religieux, pour les Anicinabek, ne sont pas des savoirs établis et valables de génération en génération. Ils sont sujets à caution si des changements imprévisibles ont lieu (colonisation, invasion des territoires, baisse des ressources fauniques, etc.). Comme nous l'avons dit plus haut, la philosophie anicinabe implique de tirer les conséquences, pratiques et morales, des faits qu'on observe afin d'agir au mieux pour retrouver un bien-être. Le chamanisme avait été remis en question par de gros changements et par le catholicisme, mais quand le catholicisme lui-même n'a plus fait ses preuves, alors il fallait rediscuter du bien-fondé du chamanisme. Ainsi en témoignent les propos de deux aînés:

C'était étrange à quel point les Anicinabek pouvaient avoir du pouvoir dans le temps. Ils avaient de grands pouvoirs. C'est parce qu'il y a un Dieu, ce n'est pas par le Diable. C'est vrai que Dieu est partout. C'est Dieu qui a donné ce pouvoir-là aux Anicinabek. [Moi] Pourquoi ce pouvoir a-t-il disparu? [Réponse] C'est ceux qui ont apporté la religion. Une autre religion que celle qu'on avait avant. On a perdu notre coutume alors, depuis qu'ils sont là. (Pikogan, $1^{\text {er }}$ mai 1996)

Ce témoignage montre que les aînés d'aujourd'hui interprètent la croyance en Dieu comme précédant l'arrivée des missionnaires. Cela permet à la fois de comprendre, en tirant les conséquences de l'histoire, pour-

21. Si les savoirs écologiques peuvent être maîtrisés, puisqu'ils sont issus d'une longue démarche expérimentale (l'observation empirique accumulée par les Anicinabek au cours des siècles) et font partie du monde visible (physique), il n'en est pas de même pour les connaissances métaphysiques. Seuls les chamans, qui avaient suivi de très durs apprentissages, étaient considérés comme capables de comprendre la réalité du monde invisible. Les autres devaient se contenter de spéculer sur le sujet. 
quoi les ancêtres s'étaient convertis, tout en expliquant que le système de croyances traditionnel était, en fait, valable.

Est-ce que des rites collectifs sont venus remplacer les rites catholiques qui ont été abandonnés? Et si oui, que révèlent-ils de l'éthique, ou de la cosmologie, ou du sens anicinabe de la communauté ? En guise de première constatation, on peut remarquer qu'on ne peut plus guère identifier de rites collectifs religieux dans lesquels se reconnaîtrait et auxquels participerait une majorité d'Anicinabek. En effet, de nos jours, le paysage religieux est très diversifié chez les Anicinabek, comme chez les autres Amérindiens d'ailleurs, les uns se disant toujours catholiques (qu'ils pratiquent ou non), d'autres se disant plutôt pentecôtistes, d'autres encore adeptes de la spiritualité panindienne (Bousquet 2007). D'autres enfin veulent retourner au chamanisme. Comme deuxième constatation, notons que la période de l'année pendant laquelle a lieu le rassemblement spirituel de Kitigan Zibi, créé en 1996, correspond aux trois premiers jours du mois d'août, soit la même période que celle des journées de Kateri. Le fait n'est sans doute pas une coïncidence: à ses débuts, le rassemblement spirituel, qui promouvait la paix et la fraternité entre les peuples, avait pour maître-mot la réconciliation autour de l'expérience de la colonisation, la dénonciation et la guérison des maux engendrés par cette colonisation et enfin la nécessité d'un retour à une spiritualité proche de la Terre-Mère ${ }^{22}$. Comme dernière constatation, il faut préciser que les événements collectifs anicinabek n'ont pas du tout éliminé le religieux: les makocanak, les pow-wows, les festivals sont généralement ouverts par des prières. Mais au lieu que ces prières soient nécessairement prononcées par un prêtre catholique, elles seront dites par un ou une aînée. Il n'est pas exclu, loin de là, que l'aîné en question soit catholique ou pentecôtiste et adresse la prière à Jésus. Mais tant que la prière est dite dans la langue anicinabe et par un ou une Anicinabe, cela n'a guère d'importance. L'important est qu'un Anicinabe occupe la place centrale dans la performance.

L'éthique anicinabe ne semble pas avoir fondamentalement changé depuis le passage à la sédentarité. Le manque de preuves pratiques, dans la vie moderne, qu'un système religieux fonctionne mieux que les autres

22. Un rassemblement spirituel dure en général deux ou trois jours. Il consiste en des prières communes à des moments précis de la journée (cérémonies de lever du soleil), des ateliers de réflexion, des rites en petits groupes (tentes à sudation) et des moments festifs (repas, danses). J'ai abondamment décrit la première occurrence du rassemblement spirituel de Kitigan Zibi dans ma thèse de doctorat, au chapitre VIII (Bousquet 2001). 
pour tout le monde permet à chacun d'expérimenter diverses croyances. Ce qui a fait ses preuves, en revanche, est la confiance en la tradition. Dans leur histoire, les Anicinabek identifient des ruptures avec cette tradition et l'une des plus grandes ruptures est l'ingérence de l'Église catholique dans leur mode de vie et de pensée. Mais si le retour aux sources est prôné par tous et toutes, les moyens pour y parvenir ne sont pas si évidents que ça. En effet, la première tendance serait d'abandonner le catholicisme, responsable de la perte culturelle et d'autres maux. Mais, pour celles et ceux qui continuent à s'identifier comme catholiques, le catholicisme fait partie des traditions puisque les ancêtres, qui avaient une mentalité anicinabe jugée plus "authentique» qu'aujourd'hui (du moins selon les Anicinabek euxmêmes), y ont adhéré. Le chamanisme, lui, est considéré comme le plus «traditionnel » et pourtant les ancêtres, meilleurs détenteurs de la tradition et références suprêmes en la matière, en avaient abandonné la plupart des pratiques. Quant à la spiritualité panindienne, elle n'était pas pratiquée par les ancêtres comme telle, mais elle est une création amérindienne et elle s'inspire d'éléments traditionnels. Le problème, pour ceux et celles qui refusent de s'y reconnaître, est que cette spiritualité amalgame des éléments issus de cultures très différentes, particulièrement, disent les Anicinabek, des cultures de l'Ouest canadien. Pour ses adhérents, peu importe que tout ne vienne pas de la culture locale, tant qu'il s'agit d'un système religieux dont les autochtones ont le contrôle. En résumé, si tout le monde s'accorde sur le bien-fondé social, voire l'absolue nécessité de renouer avec ses racines pour un bien-être présent et futur de tous les Anicinabek, l'identification des processus pour y revenir, notamment au point de vue religieux, ne fait pas consensus.

Le cœur des rites collectifs reste le bien-être de la communauté: «Ensemble, on est plus forts» (homme de 54 ans, Pikogan, janvier 2009). Les motifs pour en exécuter un ont peu changé: le nœud central, qu'étaient le rapport au territoire et les relations avec les animaux, est encore très présent, mais n'est plus forcément axé sur la réussite des activités cynégétiques et halieutiques. Les Anicinabek parlent plutôt de prière pour la protection et la sauvegarde de la Terre-Mère, ce qu'on entend par exemple couramment dans les discours prononcés au micro lors des pow-wows, pendant les pauses entre les séries de chants au tambour. Également, un motif est devenu essentiel: la notion de guérison, en particulier des traumatismes sociaux et de leurs conséquences dramatiques (abus d'alcool et de drogues, violence familiale, etc.), imputés au programme d'assimilation du gouvernement canadien et à sa mise en œuvre par les agents des Affaires 
indiennes et par les missionnaires. La prière, même catholique, peut contribuer à restaurer l'ordre sociocosmique mis à mal, entre autres, par l'évangélisation.

Quels sont donc ces rites collectifs? Si je prête attention aux éléments constituants des fêtes collectives catholiques dont j'ai parlé et que les gens regrettent d'avoir perdues (non pour le contenu catholique, comme je l'ai dit, mais pour ce que les Anicinabek y avaient investi), aucun rite ne remplace strictement les grands rites catholiques. Mais de nombreux rites comprennent plusieurs de ces éléments. Par exemple, les marches organisées par les conseils de bande comportent l'aspect processionnel. Elles sont ouvertes par la prière d'un ou d'une aînée et se terminent généralement par un discours du chef et par un pique-nique communautaire. Ces marches sont des démonstrations politiques et sociales et ont toutes pour point commun la "guérison" de quelque chose: lutte contre le racisme, engagement pour la sobriété et contre les drogues, dénonciation de la violence, guérison des blessures causées par les pensionnats indiens, etc. Ce ne sont plus les bannières des paroisses que les gens portent, mais le drapeau de la communauté et des affiches couvertes de slogans rappelant le but de la marche. Il s'agit donc de l'opposé d'une mise en scène de l'ordre colonial: les Anicinabek mettent plutôt en scène la prise en charge de leurs problèmes par eux-mêmes, de leur volonté de contrôle de leur destinée. Les rubans, eux, font encore très souvent partie du décor et de l'arsenal d'offrandes dans les rituels, notamment les rituels panindiens. Citons un exemple du début des années 1990, comparable à ce que j'ai pu observer au début des années 2000: "The lodge is decorated with ribbon offerings, tobacco tied in cloth of the four sacred colors, and bunches of sage » (Dufrene 1990, 128). Les rubans sont accrochés au bout de bâtons ou portés sur les vêtements, lors d'occasions de célébrations collectives, par exemple autour d'un défunt le jour des funérailles ou dans les rassemblements spirituels. Enfin, les Anicinabek font encore parfois des décors de feuillage, sur les sites de pow-wows, pour marquer l'abri des tambourineurs, mais ils utilisent également d'autres styles de décors pour montrer clairement la nature amérindienne du lieu et de la performance rituelle, notamment par l'érection de tipis. Ces décors ne montrent plus les «espaces de catholicité ", mais viennent rappeler que les terres où se déroulent ces rites, qu'on soit en forêt, dans une réserve ou en milieu urbain, sont des terres autochtones: au Québec, les Anicinabek n’ont jamais cédé leurs territoires. 


\section{Conclusion}

De nos jours, s'il existe toujours des rites collectifs religieux chez les Anicinabek, rassemblant des gens autour d'un système de croyances, on ne peut plus qualifier ces rites de «communautaires »: les rassemblements pentecôtistes ou panindiens, qui peuvent réunir jusqu'à 10 , voire $20 \%$ d'une communauté anicinabe, ne peuvent prétendre au qualificatif de fédérateurs, contrairement aux grands rites catholiques d'antan, auxquels tout le monde assistait, et contrairement aux grands rites chamaniques. Seuls les rites politiques contemporains, axés sur la guérison ou la dénonciation d'injustices, ont, au début du $\mathrm{XxI}^{\mathrm{e}}$ siècle, le même pouvoir de mise en scène et de promotion de l'ordre social alors recherché. La référence ultime pour agir au mieux, pour choisir les bonnes manières de faire les choses est sans conteste ce que les Anicinabek appellent «la tradition ", c'est-à-dire la façon de vivre et de penser des ancêtres. Mais les savoirs religieux entrent dans une catégorie particulière de la tradition: en effet, ces savoirs se révèlent à la lumière des leçons qu'on peut tirer du passé, des événements et des décisions prises pour y faire face. Or, depuis la colonisation et l'adhésion au catholicisme, au XIX ${ }^{\mathrm{e}}$ siècle, puis depuis la sédentarisation (concomitante de la période du pensionnat), qui n'a fait qu'aggraver les problèmes surgis avec la colonisation et l'envahissement des territoires, les conséquences des décisions accumulées ont amené les Anicinabek à dégager des enseignements. Ces enseignements leur permettent de s'adapter, de continuer à vivre aussi bien dans le monde naturel qu'en relation avec le monde surnaturel et finalement de prendre en charge les blessures engendrées par ce passé. Agir au mieux, de nos jours, chez les Anicinabek, c'est défendre les valeurs, la culture et les intérêts des Anicinabek et des autres autochtones.

Il serait difficile de nier que, jusque dans les années 1980, voire 1990, les Anicinabek pouvaient faire montre de ferveur et de foi lors des performances des grandes fêtes catholiques, parce qu'il est impossible de mesurer le niveau de leur adhésion et qu'en outre ce niveau devait probablement varier selon les individus. L'implantation de ces fêtes en milieu amérindien était destinée à créer de fortes impressions sur les fidèles et à créer une identité catholique amérindienne. Elle a connu une certaine réussite: le catholicisme fait toujours partie des systèmes de référence et un certain nombre de ses éléments se sont incorporés dans les manières de penser. 
Mais, dans l'ordre sociocosmique, les prêtres ne sont plus des leaders ${ }^{23}$ : les personnes anicinabek d'expérience, que sont souvent les aînés, dont on reconnaissait le savoir et les compétences, sont redevenues les leaders centraux des performances religieuses, le prêtre ou le pasteur n'étant invités, quand c'est le cas, qu'à remplir un strict rôle de prestataires de services.

Il est certain que les affaires de pédophilie et les dénonciations des abus commis dans les pensionnats indiens ont joué un rôle majeur dans la remise en question du pouvoir et de l'influence de l'Église catholique. Ainsi en témoigne un ancien chef de la communauté anicinabe de Kitcisakik:

L'action d'une minorité a fait mal à la majorité des représentants de l'Église, le chemin qui mène au pardon sera long et douloureux, beaucoup d'entre nous qui ont été abusés par des prêtres pédophiles n'entendront jamais ces prêtres venir nous dire: "Je m'excuse". Je suis toujours à la recherche des mots qui pourraient me permettre de faire la paix avec l'Église, des mots qui me permettront de faire à nouveau confiance aux hommes et femmes de Dieu, des mots qui pourraient me faire dire avec sérénité: "Je pardonne». Un jour peut-être. (Jimmy Papatie, Kitcisakik, 3 mai 2009)24

Mais comme le montre le discours de monsieur Papatie, les Anicinabek ne sont pas contre l'idée d'une réconciliation. Ils ont pris conscience que leurs croyances religieuses, autant que leur volonté de conserver leurs propres manières de faire, constituent des droits à défendre (par exemple dans le contexte du multiculturalisme canadien et de la Charte des droits et libertés), ce qui change leur façon de les aborder. Aujourd'hui, c'est essentiellement sur le terrain du politique qu'ont lieu les grands actes collectifs ritualisés à caractère fédérateur, parce que les leçons tirées du passé ont montré que c'est sur ce terrain-là que peut se négocier, actuellement, une bonne moralité commune.

23. Si les prêtres catholiques ne sont plus des leaders en général, il demeure que certains d'entre eux peuvent en être s'ils ont un charisme personnel (voir Bousquet 2009).

24. Réponse à l'article de Alain Crevier, «Phil Fontaine et Benoît XVI : rendez-vous historique?», 28 avril 2009, sur le site de Radio-Canada: <http://www.radio-canada. ca/nouvelles/carnets/2009/04/28/119464.shtml ? auteur=2278>, page consultée en juin 2013. 


\section{Références}

Anonyme, (septembre 1965), "Kateri Tekakwitha ", Vie Indienne, Organe des Indiens d'expression française, vol. 3/3, Montréal, Commission oblate des œuvres indiennes et esquimaudes, p. 3.

Arnaud, C. o.m.i. (février 1877), «Mission montagnaise du Lac St Jean", Annales de la propagation de la foi pour la province de Québec, Premier numéro, Montréal, Des presses à vapeur de J. A. Plinguet, p. 24-34.

Bousquet, M.-P. (2001), "Quand nous vivions dans le bois", le changement spatial et sa dimension générationnelle: l'exemple des Algonquins $d u$ Canada, Thèse de doctorat en ethnologie, Université de Paris $\mathrm{X}$-Nanterre (France), et Ph.D. en anthropologie, Université Laval (Québec).

(2005), «La production d'un réseau de sur-parenté: histoire de l'alcool et désintoxication chez les Algonquins ", Drogues, santé et société, 4/1, p. 64-85.

(2007), «Catholicisme, pentecôtisme et spiritualité traditionnelle? Les choix religieux contemporains chez les Algonquins du Québec ", dans C. Gélinas et G. Teasdale, dir., Les systèmes religieux amérindiens et inuit: perspectives historiques et contemporaines, Québec/Paris, Muséologie In-Situ/L'Harmattan, p. 155-166.

(2008), "A Question of Emotions and a Matter of Respect: Interpreting Conversion to Catholicism Among Quebec Algonquins ", dans K. S. Hele et R. Darnell, dir., Papers of the Thirty-Ninth Algonquian Conference, London, University of Western Ontario, p. 52-71.

(2009), "Régler ses conflits dans un cadre spirituel: pouvoir, réparation et systèmes religieux chez les Anicinabek du Québec ", Criminologie, 42/2, p. 53-82.

(2012a), "Why did the Catholic Cult of Saints not Function among the Algonquians? ", dans K. S. Hele et J. R. VAlentine, dir., Papers of the 40th Algonquian Conference, Albany, State University of New York Press, p. 59-78.

(2012b), " "On a juste besoin d'être aimés": les bases de la tolérance religieuse chez les Anicinabek (Algonquins) du Québec", dans Dynamiques religieuses des Autochtones des Amériques/Religious Dynamics of Indigenous People of the Americas, M.-P. BousQueT et R. Crépeau, dir., Paris, Karthala (Hommes et sociétés), p. 243-270. 
Bousquet, M.-P., et Morissette, A., (2008), «Inscrire la mémoire semi-nomade dans l'actualité sédentaire. Les églises de Pikogan et de Manawan ", Archives de sciences sociales des religions, 141, p. 9-32.

Clair, M. (2006), "Entre vision et audition: la lumière dans les missions iroquoises du $17^{\mathrm{e}}$ siècle ", Anthropologie et Sociétés, 30/3, p. 71-92.

Conkling, R. (1974), "Legitimacy and Conversion in Social Change: The Case of French Missionaries and the Northeastern Algonkian ", Ethnobistory, 21/1, p. 1-24.

Cuoq, J.-A. (1893), "Anotc Kekon", Proceedings and Transactions of the Royal Society of Canada, 11/1, p. 137-179.

DelâGE, D. (1991), "La religion dans l'alliance franco-amérindienne ", Anthropologie et Sociétés, 15/1, p. 55-87.

Dufrene, P. (1990), «Utilizing the Arts for Healing from a Native American Perspective: Implications for Creative Arts Therapies, Canadian Journal of Native Studies, 10, p. 121-131.

Fortin, J. o.m.i. (1992), Coup d'œil sur le monde merveilleux des Montagnais de la Côte-Nord, Sept-Îles, Institut Culturel et Éducatif Montagnais (ICEM).

Fourchard, L. (1999), "Naissance du baroque colonial. Les cérémonies catholiques à Ouagadougou 1900-1945 », dans O. Goerg, dir., Fêtes urbaines en Afrique: espaces, identités et pouvoirs, Paris, Karthala, p. $149-165$.

Gagnon, D. (2005), "Les Mamit Innuat et la dévotion à sainte Anne. Un exemple de l'inadéquation des concepts de tradition et de modernité dans l'étude du métissage religieux", Globe. Revue internationale d'études québécoises, 8/1, p. 135-153.

(2007), «Sainte Anne et le pouvoir manitushiun: l'inversion de la cosmologie mamit innuat dans le contexte de la sédentarisation ", dans F. LAugrand et J. Oosten, dir., La nature des esprits dans les cosmologies autochtones/Nature of Spirits in Aboriginal Cosmologies, Québec, Presses de l'Université Laval, p. 449-477.

Gallat-Morin, E. (2001), "La musique dans les rues de la NouvelleFrance ", Les cahiers de la société québécoise de recherche en musique, 5/1-2, p. 45-51.

GÉLINAS, C. (2003), «Les missions catholiques chez les Atikamekw (18371940): manifestations de foi et d'esprit pratique? ", SCHEC, Études d'histoire religieuse, 69, p. 83-99. 
Gougeon, J. P. o.m.i, (1880), «Mission de Témiskaming», Annales de la propagation de la foi pour la province de Québec, n ${ }^{\circ}$, Montréal, Cie d'imprimerie canadienne, p. 220-231.

Hallowell, A. I. (1926), "Bear Ceremonialism in the Northern Hemisphere », American Anthropologist, 28/1, p. 1-174.

Hardy, R. (2007), «Regards sur la construction de la culture catholique québécoise au XIX ${ }^{\mathrm{e}}$ siècle ", The Canadian Historical Review, 88/1, p. 7-40.

LACASSE, Z. o.m.i. (1881), «Lettre du R.P. Lacasse, O.M.I. à l'Aumônier de l'Archevêché de Québec, sur la visite de Sa Grandeur Mgr Dom. Racine, Évêque de Chicoutimi, à la Mission Montagnaise du Lac St-Jean ", ", Annales de la propagation de la foi pour la province de Québec, $\mathrm{n}^{\circ} 13$, Montréal, Cie d'imprimerie canadienne, p. 65-70.

Lamothe, A. (2005) [1977], Prêtre et shaman à Nutashquan [enregistrement vidéo], Kanapikuesht mak Kamanitushit réalisation, Montréal, Ateliers audiovisuels du Québec.

Laniel, A. o.m.i, (1893), «Maniwaki, 16 septembre 1892. Au Révérend Monsieur J. B. Proulx, Vice-Recteur de l'Université Laval, Montréal ", Annales de la propagation de la foi pour la province de Québec, $\mathrm{n}^{\circ} 49$, Montréal, Cie d'imprimerie canadienne, p. 299-317.

MacPherson, J. T. (1930), An Ethnological Study of the Abitibi Indians, Musée canadien des civilisations, bibliothèque, document d'ethnologie III-G-38M, miméo.

Martel, L.-P. o.m.i. (1934), "On en parlera longtemps!... », L’Apostolat, 5, p. $183-186$.

Moreno-Navarro, I. (1986), «Religiosité populaire andalouse et catholicisme ", Social Compass, 33/4, p. 437-455.

Morrison, K. M. (1986), "Montagnais Missionization in Early New France: The Syncretic Imperative ", American Indian Culture and Research Journal, 10/3, p. 1-23.

(1990), «Baptism and Alliance: The Symbolic Mediations of Religious Syncretism », Ethnohistory, 37/4, p. 416-437.

Pannabecker, R. K. (1996), "Tastily Bound with Ribands": Ribbonbordered Dress of the Great Lakes Indians, 1735-1839», Clothing and Textiles Research Journal, 14/4, p. 267-275. 
Proulx, J.-B. (juin 1882), "Mgr d'Ottawa dans les missions sauvages ", Annales de la propagation de la foi pour la province de Québec, $\mathrm{n}^{\circ} 17$, Montréal, Cie d'imprimerie canadienne, p. 109-130.

(1892), «Douze cents milles en canot d'écorce ou première visite pastorale de Mgr N. Z. Lorrain, évêque de Cythère, vicaire apostolique de Pontiac, dans ses missions sauvages du haut des rivières Ottawa et Saint-Maurice, de Waswanipi et de Mékiskan ", Annales de la propagation de la foi pour les provinces de Québec et de Montréal, $\mathrm{n}^{\circ} 48$, Montréal, Cie d'imprimerie Gerhardt-Berthiaume.

Remiggi, F. W., et Rousseau, L. (1998), dir., Atlas historique des pratiques religieuses: le sud-onest du Québec au XIXe siècle, Ottawa, Presses de l'Université d'Ottawa.

Ridington, R. (1988), «Knowledge, Power, and the Individual in Subarctic Hunting Societies », American Anthropologist, 90/1, p. 98-110.

Rigal-Cellard, B. (2005), "La Vierge est une Amérindienne: Kateri Tekakwitha, à l'extrême imitation de Jésus et de Marie ", in Bernadette Rigal-Cellard (éd.), Missions extrêmes en Amérique du Nord: des Jésuites à Raël, Bordeaux, Pleine Page, p. 124-156 (diffusion électronique dans Les classiques des sciences sociales, Université du Québec à Chicoutimi).

(2006), Kateri Tekakwitha et l'inculturation du catholicisme chez les Autochtones d'Amérique du Nord, Université du Québec à Chicoutimi, Les classiques des sciences sociales.

Riopel, M. (ca 1991), Sur les traces des robes noires, Ville-Marie, Société d'histoire du Témiscamingue, coll. Maison du Colon, $\mathrm{n}^{\circ} 3$.

(2003), «En missionnant sur les rives du Lac Témiscamingue, 1836-1886 », Encyclobec. URL: <http://www.encyclobec.ca/main.php? docid $=487>$.

Savard, R. (1996), L’Algonquin Tessouat et la fondation de Montréal. Diplomatie franco-indienne en Nouvelle-France, Montréal, L'Hexagone.

STroud, S. (2001), "À la recherche de la source des normes déontologiques », Philosophiques, 28/1, p. 151-171.

Viau, R. (1995), «L'autopsie d'un contact », dans O. Vincent, dir., Histoire de l'Abitibi-Témiscamingue, Québec, IQRC, p. 125-159.

Walsh, M. W. (1994), «A Three Kings Pageant at Michilimackinac, 1679», Michigan Academician, 26, p. 19-27. 


\section{Résumé}

Cet article analyse la performance de rituels catholiques communautaires, comprenant des processions et des bénédictions, chez les Anicinabek (Algonquins). Il avance que leur étude donne à voir, dans le temps, l'éthique anicinabe. En effet, la période retenue, entre les années 1950 et 2000, correspond à des changements sociaux qui ont bouleversé l'ordre sociocosmique anicinabe. En portant attention à leur déroulement, à ce qu'ils mettent en scène du corps social, des relations avec le clergé et avec le surnaturel, nous postulons que ces rituels révèlent tant les transformations de l'imaginaire social et cosmologique anicinabe qu'un système de pensée pragmatique permettant de prendre les décisions pour se comporter, être et agir au mieux. Enfin, dans le contexte actuel où le catholicisme est en nette régression, nous examinons les nouveaux rites collectifs pour constater que ceux qui promeuvent les «bonnes manières de faire" tiennent essentiellement du domaine politique, terrain où se négocie maintenant la moralité commune.

\section{Abstract}

This article analyzes the performance of Catholic communal rituals, including processions and blessings, within the Anicinabek (Algonquin), which I argue, makes visible, over time, an Anicinabe ethics. The period studied, 1950 to 2000, corresponds to a time of social change that transformed the Anicinabe socio-cosmic order. In paying attention to the conduct of communal rituals, to what they depict of the social body, of relationships with clergy and with the supernatural, we see both changes in the Anicinabe social and cosmological imaginary, and a pragmatic system of thought that enables decision making about how to behave, to be and to act best. In the current context where Catholicism is in sharp decline, this paper examines the new collective rites and shows that those that promote "good ways of doing things" are mainly framed in the political arena, the space in which the common morality is now negotiated. 